\title{
Corneal amyloidosis caused by Leu518Pro mutation of $\beta$ ig-h3 gene
}

\author{
Koji Hirano, Yoshihiro Hotta, Keiko Fujiki, Atsushi Kanai
}

Department of Ophthalmology, Nagoya University School of Medicine K Hirano

Y Hotta

\section{Department of} Ophthalmology, Juntendo University School of Medicine Y Hotta

K Fujiki

A Kanai

Correspondence to: Koji Hirano MD, PhD, Department of

Ophthalmology, Nagoya University School of Medicine, 65 Tsuruma-cho, Showa-ku, Nagoya, Aich 466-8550, Japan

kojihira@med.nagoya-u.ac.jp

Accepted for publication 28 January 2000

\begin{abstract}
Aim-To report a Japanese family diagnosed clinically as having lattice corneal dystrophy type I (LCDI) in which a Leu518Pro mutation in the $\beta$ ig-h3 gene and not the $\mathrm{R} 124 \mathrm{C}$ mutation reported previously was found.

Methods-Molecular genetic analysis was performed on DNA extracted from peripheral leucocytes from four members (three affected and one unaffected) of a family. Exon 4 of the $\beta i g-h 3$ gene was amplified by PCR and directly sequenced. Histopathological study was performed on the corneal tissue from the proband obtained during deep lamellar keratoplasty.

Results-All the affected members were clinically diagnosed as having LCDI, and the pedigree indicated an autosomal dominant inheritance. A heterozygous single base pair transition (CTG to CCG, leucine to proline) was detected in codon 518 of the $\beta i g-h 3$ gene in the three affected members, and not in the unaffected member. No mutation was found in codon 124 . Amyloid deposits were observed between the collagen bundles of the corneal stroma and were seen to extend deep into the stroma.

Conclusion-The Leu518Pro mutated ßig-h3 forms amyloidogeneic intermediates which precipitate in the cornea and gives rise to a clinical appearance of LCDI.

(Br f Ophthalmol 2000;84:583-585)
\end{abstract}

Lattice corneal dystrophy type I (LCDI) is an autosomal dominant bilaterally symmetric corneal disorder which is characterised by numerous translucent lattice lines associated with white dots and faint haze in the superficial and middle layers of the central stroma. ${ }^{12}$ The symptoms appear during the first or second decades of life. ${ }^{12}$ This dystrophy, along with another three autosomal dominant corneal disorders, granular corneal dystrophy Groenouw type I, Avellino corneal dystrophy, and Reis-Bücklers corneal dystrophy, has been mapped to chromosome 5q31. ${ }^{3-5}$ Recent studies have shown that these four dystrophies result from the mutation of TGF- $\beta$ induced gene ( $\beta$ ig-h 3$){ }^{6}$ and a R124C mutation was detected in the LCDI in both white ${ }^{6}$ and Japanese $^{7}$ families. Recently, Endo et al found a new mutation of $\beta$ ig-h3 in a Japanese family clinically diagnosed as LCDI in which a mutation could not be found in R124 but instead a
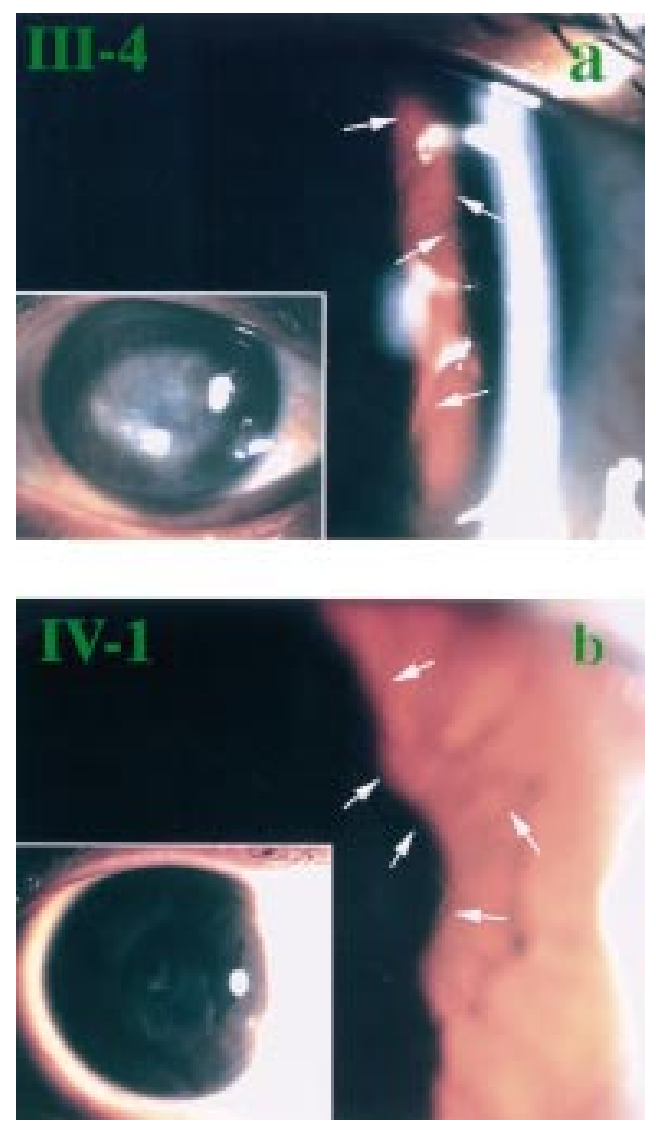

Figure 2 Slit lamp biomicroscopic findings. (a) The proband (III-4). Refractile lattice lines (arrows) are seen in the mid-peripheral area of the cornea. Inset shows the central corneal opacity. (b) Patient IV-1. Subepithelial white dots and placoid pattern of corneal opacity are seen in the inset. Refractile fine lines (arrows) can be seen.

Figure 1 Pedigree of the family. The arrow indicates the proband (III-4). III-7 and 8 are fraternal twin sisters. 

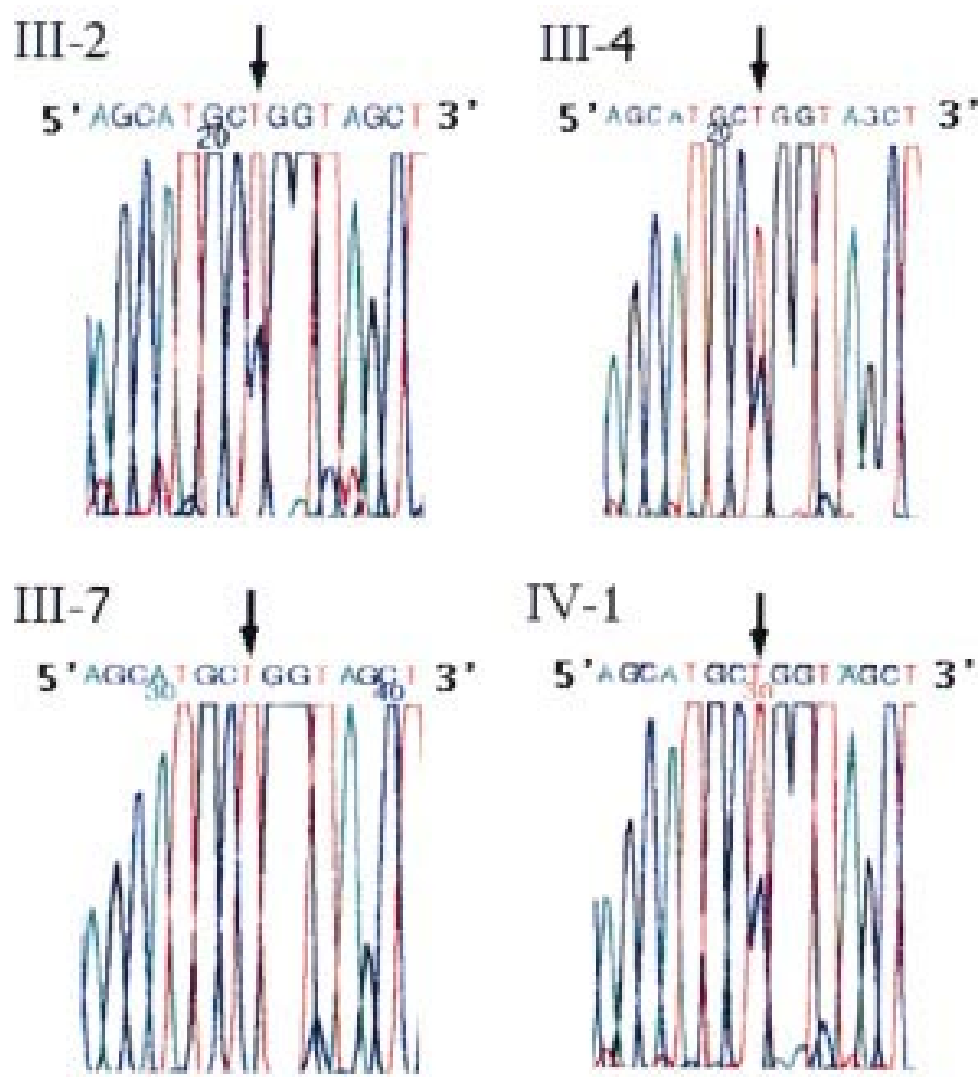

Figure 3 Nucleotide sequences of the Big-h3 gene using sense primer. Three affected individuals (III-2, III-4, IV-1) show a heterozygous single base pair transition (CTG to CCG, Leu to Pro). The proband's unaffected sister (III-7) shows wild type allele.

Leu518Pro mutation was detected. ${ }^{8}$ We present another Japanese family with the Leu518Pro mutation. Histopathological examination of a corneal specimen from one of the patients showed amyloid deposits between the collagen bundles of the corneal stroma.

\section{Patients}

The pedigree being studied is shown in Figure 1.

The proband (III-4) is 42 year old man who was referred to the department of ophthalmology of the Nagoya University Hospital from an internist. The patient complained of visual disturbance during his stay in the same hospital as an inpatient for renal failure of unknown cause. He first suffered ocular pain at 15 years of age, and noticed a visual acuity loss at 20 years of age. He was checked by an ophthalmologist and his visual acuity gradually decreased. On the first visit, his visual acuity was $20 / 200$ noncorrectable (NC) in the right eye, and 20/1000 $\mathrm{NC}$ in the left. The intraocular pressure (IOP) was $11 \mathrm{~mm} \mathrm{Hg}$ right eye and $10 \mathrm{~mm} \mathrm{Hg}$ left eye. A dense superficial corneal opacity associated with fine lattice lines in the superficial and midstromal layers was observed in the both eyes by slit lamp biomicroscopic examination (Fig 2a). The epithelium appeared smooth without any fluorescein stained regions. The lens appeared transparent but the ocular fundus was not visible through the hazy cornea.

A deep lamellar keratoplasty (DLK) was performed on his left eye (by HK), and his visual acuity improved to $20 / 60$ NC 10 days after surgery.

Patient IV-1 is a 17 year old girl who first visited an ophthalmologist because of ocular pain at 14 years of age and was followed by the same doctor. Her visual acuity gradually decreased and on her first visit to the Nagoya University Hospital, it was 20/400 (20/30 × $-5.0 \mathrm{D}=$ cyl $-1.50 \mathrm{D} \mathrm{Ax} 180)$ right eye, and $20 / 300(20 / 100 \times-3.5 \mathrm{D}=$ cyl $-3.25 \mathrm{D}$ Ax 180$)$ left eye. Her IOPs were $14 \mathrm{~mm} \mathrm{Hg}$ right eye and $10 \mathrm{~mm} \mathrm{Hg}$ left eye. Fine lattice lines were observed in the superficial and midstromal area of the corneas of both eyes. The central corneal opacity was not as dense as that of the proband, and instead, fine white dots and faint placoid pattern of superficial opacity were observed (Fig 2b). By a careful history taking, it was learned that she was a cousin of the proband. Blood examination did not show signs of renal failure or anaemia; however, hypertriglycaemia $(280 \mathrm{mg} / \mathrm{dl})$ was detected as it was in the proband $(177 \mathrm{mg} / \mathrm{dl})$.

\section{Methods}

After obtaining informed consent, molecular genetic analysis was performed. DNA was extracted from peripheral leucocytes in three affected members (III-2, III-4, and IV-1) and one unaffected family member (III-7). Exon 4 of the $\beta$ ig-h3 gene was amplified by polymerase chain reaction (PCR) and directly sequenced.

Histopathological study was performed on the corneal tissue of III-4 obtained during DLK.

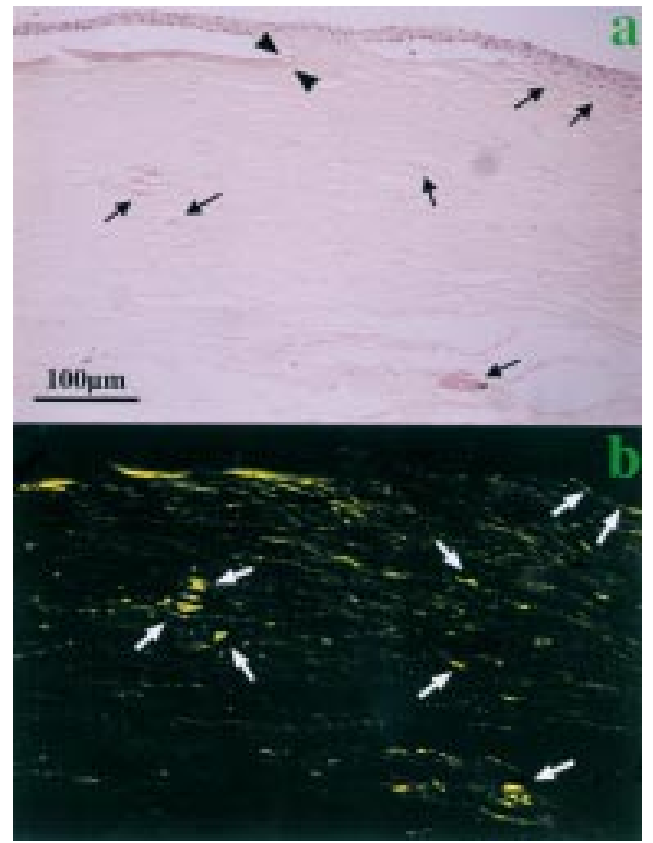

Figure 4 Histological appearance of the proband's cornea stained with congo red. (a) The epithelium varies in thickness and the interruption of Bowman's layer can be seen (arrowheads). Pink to orange coloured amyloid deposits (arrows) are seen among the bundles of the collagen fibrils. These deposits demonstrate green birefringence with a polarising filter (b, arrows) (bar $=100$ $\mu \mathrm{m})$. 
Table 1 Phenotypes of corneal dystrophies caused by the Big-h3 mutations

\begin{tabular}{ll}
\hline R124C, L518P & lattice corneal dystrophy type I \\
P501T & lattice corneal dystrophy type IIIA \\
N622H, H626R & late onset lattice corneal dystrophy \\
L527R & lattice corneal dystrophy with deep stromal opacities \\
R124S, R555W & granular corneal dystrophy (Groenouw type I) \\
R124H & Avellino corneal dystrophy \\
R124L, R555Q & Reis-Bückers dystrophy
\end{tabular}

The phenotypes of various lattice corneal dystrophies are described according to the descriptions of original articles.

\section{Results}

All three affected individuals showed a heterozygous single base pair transition (CTG to CCG, leucine to proline) (Fig 3, III-2, III-4, and IV-1). No mutation was detected in $\operatorname{Arg} 124$. The unaffected patient did not show any mutation in Leu518 (Fig 3, III-7). The nucleotide sequences were confirmed from both directions.

Histopathological observation of the removed corneal tissue showed that the epithelium varied in thickness and Bowman's layer was interrupted (Fig 4). Amyloid deposits, which were stained pink to orange by congo red, were observed between the collagen bundles and extended into the deep stroma (Fig 4a). The deposits were also observed in the subepithelial area of the epithelium. These deposits demonstrated green birefringence with a polarising filter (Fig 4b).

\section{Discussion}

Two types of corneal amyloidosis, lattice corneal dystrophy type I (LCDI) and Avellino corneal dystrophy, are known to be caused by the R124 mutation of $\beta$ ig-h3 gene, and R124 seems to be responsible for the precipitation of amyloidogenic intermediates in the corneal stroma, ${ }^{6}$ even though the codon 124 is critical in the pathogenesis of a wide range of corneal dystrophies (Table 1). ${ }^{910}$ In a recent report, however, a P501T mutation of $\beta$ ig-h3 was detected in lattice corneal dystrophy type IIIA $^{11}$ and a L527R mutation in another type of lattice corneal dystrophy with deep stromal opacities, ${ }^{12}$ instead of a R124 mutation. Table 1 lists the corneal dystrophies caused by the mutation of $\beta$ ig-h3 gene. ${ }^{6-13}$

In the family studied in this report, a L518P mutation instead of an R124 mutation of $\beta$ ig-h3 was detected in the patients with LCDI, and the unaffected member of this family revealed no mutation in the codon 518 . These results, together with those from a previous study, ${ }^{8}$ indicate that the L518P mutation of the Big-h3 gene forms amyloidogeneic intermediates that precipitate in the cornea. By careful family history taking, we learned that the family had no relatives either in Tokyo or in Joetsu where another L518 mutated LCDI family ${ }^{8}$ reported previously are from. It was thus considered that there is no connection between the two L518 mutated LCDI families.

The clinical features, obtained by the history taking from patients II- 6 , III- 2 and III- 5 as well as the two cases described, resemble quite closely those of R124C mutated LCDI. The ocular pain due to epithelial disorders which occurs in their teens is the first symptom, followed by the visual disturbance. Findings with the slit lamp were also identical to R124C mutated LCDI.

This histopathological study is the first conducted on the Leu518Pro mutated lattice dystrophic cornea, and together with the findings in R124 mutated LCDI, ${ }^{714}$ the congo red positive deposits were also detected in this dystrophy. The surgically removed corneal specimens of the proband showed an epithelium of variable thickness, an interrupted Bowman's layer, and amyloid deposits between the collagen bundles of the stroma. These findings are similar to those of LCDI reported earlier. ${ }^{1214}$ However, these deposits were distributed much deeper in the stroma compared with the R124C mutated LCDI.

Although the cause of the proband's renal failure is still unknown, hypertriglyceridaemia was found in both the proband and patient IV-1, and abnormal liver function due to fatty liver was detected in III-5. We are searching for the possibility of a general disorder derived from Leu518Pro mutation of $\beta$ ig-h3.

Supported by a grant in aid for scientific research from the Ministry of Education, Japan (K Hirano: (C) 11671732, A Kanai: (B) 05454477, (B) 0745741).

We thank Professor Yozo Miyake for invaluable suggestions during the preparation of the manuscript

1 Waring III GO, Mbekeani JN. Corneal dystrophies. In: Leibowitz HW Waring III GO eds. Corneal disorders. Clinical diagnosis and management. 2nd ed. Philadelphia: WB Saundiagnosis and managene

2 Mannis MJ, De Sousa LB, Gross RH. The stromal dystrophies. In: Krachmer JH, Mannis MJ, Holland EJ, eds. Cornea. Cornea and external disease: clinical diagnosis and management. St Louis: Mosby, 1997:1048-51.

3 Stone EM, Mathers WD, Rosenwasser GOD, et al. Three autosomal dominant corneal dystrophies map to chrosome 5q. Nat Genet 1994;6:47-51.

4 Small KW, Mullen L, Barletta J, et al. Mapping of Reis-Bücklers corneal dystrophy to chrosome 5q. Am $\mathcal{F}$ Ophthalmol 1996;121:384-90.

5 Korvatska E, Munier FL, Zografos L, et al. Delineation of a 1 -cM region on distal $5 \mathrm{q}$ containing the locus for corneal sion of the candidate genes SPARC and LOX. Eur F Hum Genet 1996;4:214-18.

6 Munier FL, Korvatska E, Djema A, et al. Kerato-epithelin mutations in four 5q31-linked corneal dystrophies. Nat Genet 1997;15:247-51.

7 Hotta Y, Fujiki K, Ono K, et al. Arg124Cys mutation of the Big-h3 gene in a Japanese family with lattice corneal dystrophy type I. Fpn $\mathcal{F}$ Ophthalmol 1998;42:450-5

8 Endo S, Thanh Ha N, Fujiki K, et al. Leu518Pro mutation of the $\beta$ ig-h3 gene causes lattice corneal dystrophy type I. Am F Ophthalmol 1999;128:104-6.

9 Stewart HS, Ridgway AE, Dixon MJ, et al. Heterogeneity ingranular corneal dystrophy: identification of three causative mutations in the TGF- 3 I ( $\beta$ ig-h3) gene-lessons for corneal amyloidogenesis. Hum Mutat 1999;14:126-32.

10 Okada $\mathrm{M}$, Yamamoto S, Tsujikawa $\mathrm{M}$, et al. Two distinct phy. Am f Ophthalmol 1998;126:535-42.

11 Yamamoto S, Okada $M$, Tsujikawa $M$, et al. A keratoepithelin ( $\beta$ ig-h3) mutation in lattice corneal dystrophy epithelin (ßig-h3) mutation in lattice cornea
type IIIA. Am f Hum Genet 1998;62:719-22.

12 Fujiki K, Hotta Y, Nakayasu K, et al. A new L527R mutation of the $\beta$ ig-h3 gene in patients with lattice corneal dystrophy with deep stromal opacities. Hum Genet 1998;103:286-9.

13 Stewart H, Black GCM, Donnai D, et al. A mutation within exon 14 of the TGFBI (BIGH3) gene on chromosome 5 q31 causes an asymmetric, late-onset form of lattice corneal dystrophy. Ophthalmology 1999;106:964-70.

14 Streeten BW, Qi M, Klintworth GK, et al. Immunolocalization of $\beta$ ig-h3 protein in 5q31-linked corneal dystrophies and normal corneas. Arch Ophthalmol 1999;117:67-75. 